\title{
Nephrotoxic Medication Exposure in U.S. Adults with Predialysis Chronic Kidney Disease: Health Services Utilization and Cost Outcomes
}

\author{
Mary Lynn Davis-Ajami, PhD; Jeffery C. Fink, MD, MS; and Jun Wu, PhD
}

\begin{abstract}
BACKGROUND: Nephrotoxic medication exposure increases risks for acute kidney injury, permanent renal function loss, and costly preventable adverse drug events. Exposure to medications associated with inducing acute tubular nephritis or tubular toxicity versus nonexposure among those with predialysis renal disease-a population vulnerable to increased risk of kidney injury-may affect health services utilization and cost outcomes. Few studies quantify nephrotoxic medication exposure in chronic kidney disease (CKD) and associated costs.
\end{abstract}

OBJECTIVE: To examine exposure to medications associated with inducing acute tubular nephritis or tubular toxicity versus nonexposure and the effect on health services utilization and cost outcomes in a nationally representative sample of adults with predialysis CKD.

METHODS: This retrospective study used Medical Expenditure Panel Survey (MEPS) household component longitudinal files (years 2006-2012; panels 11-16). Participants included 809 MEPS respondents aged $>18$ years with predialysis CKD, after excluding those participants with cancer, kidney stone, renal dialysis, or transplant procedures (approximately 14.7 million U.S. noninstitutionalized individuals). Two groups were created to evaluate the main measures: (1) participants prescribed 1 or more medications associated with risk of acute tubular nephritis and/or tubular toxicity (termed "nephrotoxic exposure") and (2) participants with nonexposure. Medications cited in published literature as associated with tubular kidney damage were used. Multivariable regression models assessed the pattern of nephrotoxic medication exposure and its effect on health services utilization and expenses.

RESULTS: Nephrotoxic medication exposure occurred in $72 \%$ of adult MEPS respondents. Of those, $47.2 \%$ and $52.8 \%$ were prescribed 1 and at least 2 nephrotoxic medications, respectively. Coexistent chronic conditions included hypertension $(72.3 \%)$, diabetes $(49.5 \%)$, coronary heart disease $(33 \%)$, arthritis (23.6\%), and chronic obstructive pulmonary disease (17.6\%). Eligible MEPS respondents aged $\geq 65$ years, from the U.S. South region, and with Charlson Comorbidity Index (CCI) score $>0$ were $75 \%$ (vs. aged $18-45$ years), $83 \%$ (vs. Northeast), and $72 \%-96 \%$ (vs. $\mathrm{CCl}=0$ ) more likely to be exposed to nephrotoxic medications. Uninsured participants showed $55 \%$ less likelihood of nephrotoxic exposure, compared with privately insured participants. Higher utilization was shown in the nephrotoxic medication exposure group (vs. nonexposure): prescription fills (52.8 vs. 26.8, $P<0.001$ ), emergency department visits (56.2 vs. 29.3 per 1,000 patient months, $P<0.001$ ), and hospitalization (51.8 vs. 23.4 per 1,000 patient months, $P<0.001)$. Unadjusted all-cause expenses were greater for the following categories: medical $(\$ 119,935$ vs. $\$ 11,462, P<0.001)$, prescription drug $(\$ 4,828$ vs. $\$ 2,816, P<0.001)$, and total health expenses ( $\$ 24,663$ vs. $\$ 14,277, P<0.001)$. Adjusted all-cause expenses were greater for total $(29.7 \%$ greater, $P=0.003)$, prescription medications $(56.6 \%$ greater, $P<0.001$ ), and medical (23.4\% greater, $P=0.036)$, but there were no differences in predialysis CKD-related utilization and expenses.
CONCLUSIONS: Increased vigilance is needed when prescribing nephrotoxic medications in predialysis CKD, particularly in patients with comorbid conditions and the elderly. Nephrotoxic medication exposure in predialysis CKD has the potential for increased health services utilization and cost outcomes.

J Manag Care Spec Pharm. 2016;22(8):959-68

Copyright $\odot 2016$, Academy of Managed Care Pharmacy. All rights reserved.

\section{What is already known about this subject}

Drug-induced kidney damage contributes to the estimated 1.5 million preventable adverse drug events that cost the United States an annualized $\$ 3.5$ billion dollars, which likely underestimates the true cost.

Few studies provide nationally representative information comparing health utilization and expenses in patients with predialysis chronic kidney disease (nephrotoxic medication exposure vs. nonexposure).

\section{What this study adds}

Nearly $72 \%$ of subjects with predialysis chronic kidney disease were prescribed nephrotoxic medications, representing 10.5 million U.S. individuals (2006-2012).

Higher health care utilization was observed in nephrotoxic medication exposure, including prescription drug use, emergency department visits, and hospitalization

Total health expenses in nephrotoxic medication exposure were nearly 30\% higher than nonexposure group.

$\mathrm{N}$ ephrotoxic medication exposure can initiate acute kidney injury (AKI) and/or potentially initiate or exacerbate chronic kidney disease (CKD)., ${ }^{1,2}$ Nephrotoxicity varies by agent and can occur after 1 dose, be dose dependent, and/or have cumulative dose effects over time from various pathogenic mechanisms (e.g., altered intraglomerular hemodynamics, tubular cell toxicity, inflammatory response, crystal nephropathy, rhabdomyloysis, and thrombotic microangiopathy). ${ }^{3-5}$ Individuals with CKD and reduced renal function are more vulnerable to toxic insults that lead to AKI, which may lead to transient or permanent reduction in glomerular 
filtration rate (GFR). ${ }^{1}$ The vulnerability of CKD patients to renal damage is concerning because overall CKD is common, increasingly affecting the elderly (e.g., incidence more than doubled between the years 2000 and 2008), ${ }^{6}$ is estimated to affect 26 million U.S. adults, ${ }^{7}$ and is frequently underreported. ${ }^{2}$ From 2007 to 2012, general U.S. population prevalence of CKD (defined by an estimated GFR $<60 \mathrm{~mL} / \mathrm{min} / 1.73 \mathrm{~m}^{2}$ or an albu$\mathrm{min} /$ creatinine ration $\geq 30 \mathrm{mg}$ per $\mathrm{gm}$ ) was $14.0 \%$, with stage 3 representing the greatest prevalence, as well as the stage with the greatest trend towards increased prevalence. ${ }^{8}$ For the year 2011, the Centers for Disease Control and Prevention reported prevalence of CKD by stage in the U.S. Veterans Affairs Health System as follows: stage $3=10.29 \%$, stage $4=0.68 \%$, and dialysis $=0.41 \% .{ }^{9}$ Preventing drug-induced nephrotoxic exposure is paramount for preserving renal function and reducing the incidence of AKI and end-stage renal disease (ESRD).

Drug-induced kidney damage contributes to the estimated 1.5 million preventable adverse drug events (ADEs) that cost the United States an annualized $\$ 3.5$ billion, which likely is an underestimate of the true cost..$^{10-12}$ Individuals with CKD, the elderly, or those with chronic disease such as diabetes, hypertension, cardiovascular disease, heart failure, and sepsis are at greater risk for ADEs that cause kidney damage. ${ }^{13}$ Harmful medication exposure among those with compromised renal function could potentially increase health care utilization and costs through increased AKI, metabolic complications, and progression to ESRD. Preexisting CKD increases the risk for AKI as much as 10 times compared with those individuals without CKD. ${ }^{14,15}$ AKI may cause CKD and if superimposed on CKD increases the risk for kidney disease progression. ${ }^{16}$ Although we know inappropriate exposure to renally excreted medications or metabolized drugs poses a risk to kidney disease progression, few studies focus on how harmful medication exposures affect cost and utilization outcomes in predialysis CKD. Additionally, although the various mechanism for, risks associated with, and outcomes from nephrotoxic medication exposure are known, few studies provide nationally representative information comparing individuals with nephrotoxic medication exposure with nonexposure in predialysis CKD, along with the associated health services utilization and costs. Further, there is little information that assesses nephrotoxic drug exposure by coexistent chronic disease in the predialysis CKD population.

A PubMed literature review using search terms ((nephrotoxic) AND medication) AND CKD) AND cost) returned zero articles, while search terms ((nephrotoxic) AND medication) AND CKD) AND utilization) returned 1 foreign study about the incidence of nephrotoxicity of iron supplementation in CKD. No studies reported health services utilization outcomes in U.S. adults with CKD exposed to a nephrotoxic agent. Another search using the search terms nephrotoxic AND medication exposure AND CKD returned 2 articles. One study reported that high nephrotoxic medication exposure in a pediatric population with normal baseline kidney function progressed to CKD in 6 months. ${ }^{17}$ The second retrospective observational study reported the incidence of AKI as a result of medication exposure among those under aged 21 years. ${ }^{18}$ The literature search did not return any literature reporting cost or health services utilization outcomes in the adult predialysis CKD population in relation to nephrotoxic medication exposure.

Preventing nephrotoxic medication exposure is challenging when prescribing pharmacotherapy for adults with predialysis CKD because of the demands of comorbid conditions, which can include the need for pain management; use of concomitant medications; and complications of advanced age, such as dehydration, dementia, and sepsis, along with other factors. Safe kidney care requires awareness of the scope of potentially nephrotoxic medication-prescribing patterns and associated outcomes among those patients with CKD.

The purpose of this study was to compare differences in health services utilization and cost outcomes between predialysis CKD patients exposed to medications associated with risk of acute tubular nephritis and/or tubular toxicity (termed "nephrotoxic") versus those prescribed any other or no medications. These differences were compared in select chronic disease groups (i.e., asthma, arthritis, chronic obstructive pulmonary disease (COPD), coronary heart disease, diabetes, hypertension, and hepatitis).

\section{Methods}

\section{Data Source}

A retrospective cross-sectional study was conducted using the Medical Expenditure Panel Survey (MEPS) household component (MEPS-HC) longitudinal files for years 2006-2012 (panels 11-16) of eligible MEPS participants aged 18 years or older diagnosed with predialysis CKD. MEPS is a subsample of the previous year's National Health Interview Survey and provides a nationally representative sample of the U.S. noninstitutionalized population. The overlapping panel design of the survey includes 5 rounds of interviews covering 2 full calendar years to collect detailed information about demographic and socioeconomic characteristics, health status, access to health care, and health care utilization and expenses. ${ }^{19}$ MEPS longitudinal data files, medical condition, and event files were accessed to identify eligible subjects and relevant outcomes.

\section{Study Population}

The study sample included eligible MEPS participants aged 18 years and older with predialysis CKD. Individuals with predialysis CKD were identified from the MEPS medical condition files using Clinical Classification Software (CCS) for International Classification of Diseases, Ninth Revision, Clinical Modification (ICD-9-CM) diagnosis and procedure categorization codes developed by the Healthcare Cost and Utilization Project, 
Agency for Healthcare Research and Quality. ${ }^{20,21}$ CCS codes collapse ICD-9-CM codes into uniform and standardized smaller numbers of clinically meaningful categories. The following CCS codes were used: 156 (nephritis, nephrosis, renal sclerosis), 157 (acute and unspecified renal failure), 158 (chronic kidney disease), and 161 (other diseases of the kidneys and ureters). Individuals with diagnoses for kidney stones, renal dialysis, kidney or other organ transplant, cancer, or antineoplastic agent use were excluded (see Appendix, available in online article). Eligible participants (aged $\geq 18$ years) were defined as participating in the entire 2-year panel survey period and completing all 5 interview rounds as evidenced by having longitudinal weights greater than zero. Then, the following 2 groups were created: (1) those participants who were prescribed medications associated with inducing acute tubular nephritis and/or tubular toxicity and (2) those participants with any other or no prescription medications.

This study limited the term "nephrotoxic drug exposure" to 1 type of underlying mechanism: acute tubular nephritis and/or tubular toxicity. To classify medications as nephrotoxic, the medication had to be referred to in the literature as potentially associated with inducing acute tubular nephritis and/or tubular toxicity. ${ }^{3,4,22}$ Excluded were drugs whose potential for nephrotoxicity arose from a different mechanism (e.g., thrombotic microangiopathy or rhabdomyolysis) or drugs causing idiosyncratic acute interstitial nephritis (e.g., hypersensitivity reactions). Since no definitive list exists delineating nephrotoxic medications significantly associated with tubular nephritis and/or tubular toxicity, the medications included in this study were intended to be representative but may not be exhaustive. An "exposure" was defined as an individually prescribed medication identified from the MEPS prescribed medication files as nephrotoxic, using the MEPS variable "MULTUM" signifying the Cerner Multum therapeutic drug class designations. ${ }^{23-25}$ MULTUM categories for acute tubular nephritis medications included MULTUM class designations for the following: antiinfective agents (aminoglycosides, cephalosporin, quinolones, penicillin, all beta-lactamase inhibitors, and sulfonamides); diuretics (loop and thiazide); nonsteroidal anti-inflammatory drugs (NSAIDs); proton pump inhibitors; and others (amphotericin B, allopurinol, phenytoin, and ranitidine). Categories for tubular toxicity agents included antiretroviral, radiological agents (contrast dye), and other (zoledronate).

Comorbid chronic diseases were identified from MEPS medical condition files and included the following diagnostic categories and corresponding CCS numeric codes: asthma (128), arthritis $(202,203)$, COPD (127), coronary heart disease $(101,108)$, diabetes $(49,50)$, hypertension $(98,99)$, and hepatitis (6). ${ }^{21}$ The Deyo version of the CCI was constructed using the 3-digit ICD-9-CM codes from the medical condition files and calculated as a measure of overall comorbidity. ${ }^{26,27}$

\section{FIGURE 1 Study Inclusion Criteria}

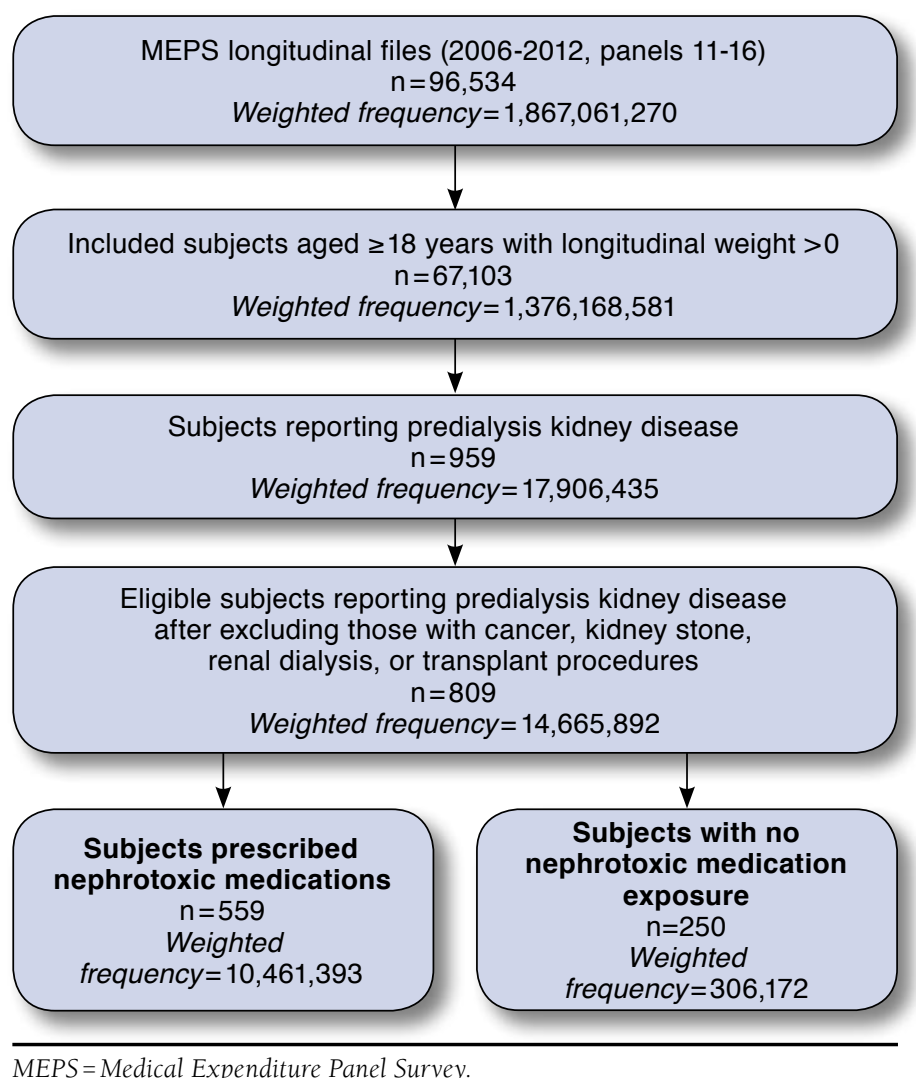

MEPS = Medical Expenditure Panel Survey.

\section{Outcomes Measure}

Economic outcomes were measured as annualized health care expenses per subject. Annualized expenses were grouped as medical expenses and prescription drug expenses. Total health care expenses were defined as the sum of medical and prescription drug expenses. Medical expenses were identified from MEPS medical event files and summed; each included MEPS participant's total expenses from office-based, outpatient, and emergency department (ED) visits and in-patient hospitalizations. Prescription medication expenses were identified from the prescribed medicines files. Total prescription medication expenses summed out-of-pocket payments and amounts paid by third-party payers. All expenses were inflated to the year 2012 using the Consumer Price Index. ${ }^{28,29}$

Two types of health care expenses were identified from the event files: all-cause expenses and CKD-related expenses. Allcause expenses combined any medical and prescription drug expenses associated with all diagnosed medical conditions per individual during the 2-year MEPS interview time frame. Predialysis CKD-related expenses were expenses for health services or treatments linked specifically with diagnosis codes 
TABLE 1 Characteristics of Adult Predialysis CKD Study Population by Medications Associated with Exposure and Nonexposure to Nephrotic Medications ${ }^{\mathrm{a}}$

\begin{tabular}{|c|c|c|c|c|c|c|c|}
\hline Variable & $\begin{array}{c}\text { Nephrotoxic } \\
\text { Exposure } \\
(\mathbf{n}=559, \text { weighted } \\
\mathbf{n}=10,461,393) \\
\mathbf{n}(\text { weighted } \%)\end{array}$ & $\begin{array}{c}\text { Nonexposure } \\
(\mathrm{n}=250, \text { weighted } \\
\mathbf{n}=306,172) \\
\mathrm{n}(\text { weighted } \%)\end{array}$ & $P$ Value & Variable & $\begin{array}{c}\text { Nephrotoxic } \\
\text { Exposure } \\
(\mathbf{n}=559, \text { weighted } \\
\mathbf{n}=10,461,393) \\
\mathbf{n} \text { (weighted } \%)\end{array}$ & $\begin{array}{c}\text { Nonexposure } \\
(\mathbf{n}=250, \text { weighted } \\
\mathbf{n}=306,172) \\
\mathrm{n}(\text { weighted \%) }\end{array}$ & $P$ Value \\
\hline \multicolumn{3}{|l|}{ Age, years } & \multirow[t]{4}{*}{0.003} & \multicolumn{3}{|l|}{ Poverty line } & \multirow[t]{5}{*}{0.901} \\
\hline $18-45$ & $94(16.8)$ & $71 \quad(27.2)$ & & Poor & $174(25.1)$ & $79(23.6)$ & \\
\hline $46-64$ & $204(33.5)$ & $95(36.9)$ & & Low income & $121 \quad(20.9)$ & $47(19.0)$ & \\
\hline $65+$ & $261(49.7)$ & $84(35.9)$ & & Middle income & $148 \quad(27.3)$ & $71 \quad(29.9)$ & \\
\hline \multicolumn{3}{|l|}{ Region } & \multirow[t]{5}{*}{0.092} & High income & $116(26.7)$ & $53(27.5)$ & \\
\hline Northeast & $86(15.9)$ & $43(24.2)$ & & \multicolumn{3}{|l|}{ Perceived health status } & \multirow[t]{4}{*}{0.073} \\
\hline Midwest & $113(21.8)$ & $40(20.1)$ & & \multirow{3}{*}{$\begin{array}{l}\text { Excellent/very good } \\
\text { Good } \\
\text { Fair/poor }\end{array}$} & $88 \quad(19.4)$ & $52(24.3)$ & \\
\hline South & $239(40.4)$ & $106(36.4)$ & & & $143(25.3)$ & $76 \quad(32.1)$ & \\
\hline West & 121 (21.9) & $61 \quad(19.3)$ & & & $326(55.2)$ & $122(43.5)$ & \\
\hline \multicolumn{3}{|l|}{ Sex } & \multirow{4}{*}{0.126} & \multicolumn{3}{|c|}{ Perceived mental health status } & \multirow[t]{4}{*}{0.161} \\
\hline Male & $231(41.5)$ & $113(47.9)$ & & Excellent/very good & $165(52.2)$ & 69 (49.9) & \\
\hline Female & $328(58.5)$ & $58(52.1)$ & & Good & $131 \quad(35.0)$ & $54(30.2)$ & \\
\hline \multirow{2}{*}{\multicolumn{3}{|c|}{ Race }} & & Fair/poor & $58(12.8)$ & 37 (19.9) & \\
\hline White & & & \multirow{4}{*}{0.687} & \multicolumn{3}{|l|}{ Insurance coverage } & \multirow[t]{4}{*}{0.008} \\
\hline & $280 \quad(61.4)$ & $131 \quad(64.0)$ & & Private & $238 \quad(51.2)$ & $111 \quad(53.7)$ & \\
\hline & $135(16.0)$ & $58(15.8)$ & & Public & $293 \quad(45.3)$ & $110(36.6)$ & \\
\hline Other & $144(22.6)$ & $61 \quad(20.1)$ & & Uninsured & $28 \quad(3.5)$ & $29 \quad(9.7)$ & \\
\hline \multicolumn{3}{|l|}{ Body mass index } & \multirow[t]{4}{*}{0.207} & \multicolumn{3}{|c|}{ Charlson Comorbidity Index } & \multirow[t]{4}{*}{0.008} \\
\hline Underweight to normal & $145 \quad(27.8)$ & $91 \quad(35.4)$ & & 0 & $289 \quad(51.6)$ & $174(69.8)$ & \\
\hline Overweight & $179(30.4)$ & $71(28.6)$ & & 1 & $127(22.1)$ & $43 \quad(15.9)$ & \\
\hline Obese & $235(41.7)$ & $88(36.0)$ & & $2+$ & $143(26.3)$ & $33(14.2)$ & \\
\hline \multicolumn{3}{|l|}{ Marital status } & \multirow[t]{3}{*}{0.114} & Chronic disease & & & \\
\hline Yes & $237(43.5)$ & $121(50.9)$ & & Hypertension & $432(77.2)$ & $150 \quad(60.1)$ & $<0.001$ \\
\hline No & $322(56.5)$ & $129(49.1)$ & & Diabetes & $283(50.6)$ & $117(46.8)$ & 0.1427 \\
\hline \multicolumn{3}{|l|}{ Highest degree } & \multirow[t]{4}{*}{0.342} & $\begin{array}{l}\text { CHD } \\
\text { Arthritis }\end{array}$ & $\begin{array}{ll}218 & (39.0) \\
146 & (26.1)\end{array}$ & $\begin{array}{ll}48 & (17.9) \\
43 & (174)\end{array}$ & $\frac{<0.001}{0.054}$ \\
\hline High school & $274(52.5)$ & $45.6 \quad(108)$ & & COPD & $125(22.4)$ & $14 \quad(5.7)$ & $<0.001$ \\
\hline College & $66(13.6)$ & $33(15.2)$ & & Asthma & $80(14.3)$ & $21 \quad(8.3)$ & 0.117 \\
\hline No degree/other & $219(33.8)$ & $109(39.1)$ & & Hepatitis & $9 \quad(1.7)$ & $2 \quad(0.8)$ & 0.343 \\
\hline
\end{tabular}

${ }^{a}$ Nephrotic exposure is defined as medications associated with inducing acute tubular nephritis and/or tubular toxicity. Study population is from MEPS, 2006-2012 $(n=809$, weighted $n=14,665,892)$.

$C K D=$ chronic kidney disease $C H D=$ coronary heart disease; $C O P D=$ chronic obstructive pulmonary disease; MEPS= Medical Expenditure Panel Survey.

for predialysis CKD. For medical utilization, predialysis CKDrelated expenses were identified by CCS codes from medical event-medical condition linked files. For prescription drugs, CKD-related medications were identified from prescribed medicines-medical condition linked files.

\section{Data Analysis}

Statistical analysis used t-tests for continuous variables and chisquare tests for categorical variables to compare differences in baseline characteristics, medical utilization, and health expenses between subjects prescribed nephrotoxic medications and subjects with any other or no prescription medications. Variables for baseline characteristics with $P$ values $<0.10$ were then included in logistic regression models to identify significant factors associ- ated with the likelihood of being prescribed 1 or more nephrotoxic medication and to compare differences in health services utilization between those with a nephrotoxic medication exposure and those with no exposure. Linear regression models with log transformation examined the association between nephrotoxic medication exposure and health care expenses to address the highly skewed cost data. ${ }^{30}$ Statistical significance was determined a priori as $P<0.05$. To generalize the findings to the U.S. population, all data analyses and statistical procedures accounted for the MEPS complex survey sampling design by applying longitudinal weights, variance estimation stratum, and primary sampling unit (clustering) and were performed using SAS version 9.3 (SAS Institute, Carey, NC). 


\begin{tabular}{|c|c|c|}
\hline TABLE 2 & \multicolumn{2}{|c|}{$\begin{array}{l}\text { Factors Associated with Prescription of } \\
1 \text { or More Medications that Induced } \\
\text { Acute Tubular Nephritis and/or Tubular } \\
\text { Toxicity in Adults with Predialysis CKDa }\end{array}$} \\
\hline Independent Variable & $\begin{array}{c}\text { Likelihood of } \\
\text { Nephrotoxic Exposure } \\
\text { Odds Ratio (95\% CI) }\end{array}$ & $P$ Value \\
\hline \multicolumn{3}{|l|}{ Age (years) } \\
\hline $18-45$ & 1.00 & \\
\hline $46-64$ & $1.26(0.78-2.02)$ & 0.341 \\
\hline$\geq 65$ & $1.75(1.04-2.96)$ & 0.036 \\
\hline \multicolumn{3}{|l|}{ Perceived health status } \\
\hline Excellent to very good & 1.00 & \\
\hline Good & $0.89(0.48-1.66)$ & 0.727 \\
\hline Fair to poor & $1.40(0.84-2.32)$ & 0.198 \\
\hline \multicolumn{3}{|l|}{ Insurance } \\
\hline Private & 1.00 & \\
\hline Public & $1.06(0.67-1.68)$ & 0.793 \\
\hline Uninsured & $0.45(0.22-0.91)$ & 0.027 \\
\hline \multicolumn{3}{|l|}{ Region } \\
\hline Northeast & 1.00 & \\
\hline Midwest & $1.73(1.00-3.00)$ & 0.051 \\
\hline South & $1.83(1.18-2.84)$ & 0.007 \\
\hline West & $1.67(1.01-2.77)$ & 0.046 \\
\hline \multicolumn{3}{|c|}{ Charlson Comorbidity Index } \\
\hline 0 & 1.00 & \\
\hline 1 & $1.72(0.99-2.96)$ & 0.053 \\
\hline $2+$ & $1.96(1.09-3.54)$ & 0.025 \\
\hline \multicolumn{3}{|c|}{$\begin{array}{l}{ }^{a} \text { From MEPS, } 2006-2012(n=809, \text { weighted } n=14,665,892) \text {. } \\
C I=\text { confidence interval; } C K D=\text { chronic kidney disease; MEPS = Medical } \\
\text { Expenditure Panel Survey. }\end{array}$} \\
\hline
\end{tabular}

\section{Results}

Figure 1 illustrates how the study sample was selected: 809 eligible subjects with predialysis CKD (weighted frequency: 14,665,892 noninstitutionalized U.S. population) were identified over the 7-year period, and nearly $72 \%$ of that number were prescribed nephrotoxic medications (weighted frequency: approximates 10.5 million U.S. individuals).

Table 1 reports characteristics of the study population (nephrotoxic exposure vs. nonexposure). Those prescribed medications associated with inducing acute tubular nephritis and/or tubular toxicity showed greater proportions from subjects aged 65 years or older $(49.7 \%$ vs. $35.9 \%, P=0.003)$, covered by public insurance $(45.3 \%$ vs. $36.6 \%, P=0.008)$, and with higher comorbidity scores $(\geq 2 ; 26.3 \%$ vs. $14.2 \%$, $P=0.001)$ than those with no nephrotoxic medication exposure. The coexistent chronic diseases distribution for this predialysis CKD study population showed hypertension as the most common coexistent chronic disease (72.3\%), followed by diabetes $(49.5 \%)$, coronary heart disease $(33 \%)$, arthritis (23.6\%), and COPD (17.6\%). Table 1 presents the comparison of exposure to nephrotoxic medications versus nonexposure by coexistent chronic disease and shows greater proportions with hypertension $(77.2 \%$ vs. $60.1 \%, P<0.001)$, coronary heart diseases $(39.0 \%$ vs. $17.9 \%, P<0.001)$, and COPD $(22.4 \%$ vs. $5.7 \%, P<0.001)$

Nephrotoxic medication exposure by drug classification included 58\% diuretics, $43 \%$ proton pump inhibitors, 22\% NSAIDs, and 27\% anti-infective agents. Additionally, among those with nephrotoxic medication exposure, more than 50\% were prescribed at least 2 types of nephrotoxic medications.

Table 2 shows significant factors associated with the likelihood for nephrotoxic medication exposure. Significant factors with greater likelihood for nephrotoxic medication exposureincluded age $>65$ years (odds ratio $[\mathrm{OR}]=1.75,95 \%$ confidence interval $(\mathrm{CI})=1.04-2.96$ [vs. 18-45 years]), greater comorbidity ( $\mathrm{CCI}>1$ [vs. $\mathrm{CCI}=0$ ]: $\mathrm{OR}=1.96,95 \% \mathrm{CI}=1.09-3.54)$, and living in the South geographical region $(\mathrm{OR}=1.83,95 \% \mathrm{CI}=1.18$ 2.84 [vs. Northeast]). Individuals without health insurance coverage were $55 \%$ less likely to be prescribed nephrotoxic medications $(\mathrm{OR}=0.45,95 \% \mathrm{CI}=0.22-0.91)$ than those covered by private insurance.

Table 3 compares health service utilization patterns and health expenses in individuals prescribed nephrotoxic versus any other or no medications. Subjects prescribed medications that may induce acute tubular nephritis and/or tubular toxicity (termed "nephrotoxic") showed higher all-cause annualized health service utilization per subject, including number of prescription fills ( 52.8 vs. $26.8, P<0.001$ ), ED visits ( 56.2 vs. 29.3 per 1,000 patient months, $P<0.001$ ), and hospitalization events (51.8 vs. 23.4 per 1,000 patient months, $P<0.001$ ) than those with no nephrotoxic medications. Significant differences in allcause health expenses per subject per year were observed in the 2 groups (nephrotoxic medication exposure vs. nonexposure), including medical expenses $(\$ 19,835$ vs. $\$ 11,462, P<0.001)$, prescription drug expense $(\$ 4,828$ vs. $\$ 2,816, P<0.001)$, and total health expenses $(\$ 24,663$ vs. $\$ 14,277, P<0.001)$.

After adjusting for demographic and health-related covariates, individuals prescribed nephrotoxic medications showed 29.7\% higher all-cause total health expenses $(P=0.003), 23.4 \%$ higher all-cause medical expenses $(P=0.036)$, and $56.8 \%$ higher all-cause prescription drug expenses $(P<0.001)$ than those with no nephrotoxic medications use (Table 4). No significant differences were found in predialysis CKD-related health services utilization and total health expenses between the groups.

\section{Discussion}

This study is among the first to provide national estimates for health services utilization and expenses for those exposed to medications associated with acute tubular nephritis and/or tubular toxicity, defined as nephrotoxic versus nonexposure among a cohort of individuals with predialysis CKD. This study's findings demonstrated significant differences between 
TABLE 3 Patterns of Health Service Utilization and Health Care Expenses by Prescribed Medications Associated with Nephrotoxic Exposure in Adults with Predialysis CKD ${ }^{a}$

\begin{tabular}{|c|c|c|c|}
\hline & $\begin{array}{l}\text { Nephrotoxic Exposure } \\
(\mathrm{n}=559, \text { weighted } \\
\mathrm{n}=10,461,393)\end{array}$ & $\begin{array}{l}\text { Nonexposure } \\
(\mathrm{n}=250, \text { weighted } \\
\mathrm{n}=306,172)\end{array}$ & $P$ Value \\
\hline \multicolumn{4}{|l|}{ All-cause health service utilization, mean (SE) } \\
\hline Number of office visits & $21.2 \quad(1.3)$ & $16.1 \quad(1.8)$ & 0.029 \\
\hline Number of outpatient visits & $2.7 \quad(0.4)$ & $1.7 \quad(0.3)$ & 0.051 \\
\hline Number of prescription fills & $52.8 \quad(2.1)$ & $26.8 \quad(2.0)$ & $<0.001$ \\
\hline ED visits (per 1,000 patient months), $\mathrm{n}$ & 56.2 & 29.3 & $<0.001$ \\
\hline Hospitalization (per 1,000 patient months), $\mathrm{n}$ & 51.8 & 23.4 & $<0.001$ \\
\hline \multicolumn{4}{|c|}{ Kidney disease-related health service utilization, mean (SE) } \\
\hline Number of office visits & $9.3 \quad(1.1)$ & $9.1 \quad(1.6)$ & 0.928 \\
\hline Number of outpatient visits & $1.4 \quad(0.3)$ & $1.0 \quad(0.3)$ & 0.404 \\
\hline Number of prescriptions & $3.7 \quad(0.3)$ & $2.6 \quad(0.4)$ & 0.029 \\
\hline ED visits (per 1,000 patient months), $n$ & 8.0 & 5.2 & 0.083 \\
\hline Hospitalization (per 1,000 patient months), $\mathrm{n}$ & 10.2 & 7.0 & 0.090 \\
\hline \multicolumn{4}{|l|}{ All-cause health expenses (\$), mean (SE) } \\
\hline Total expenses & $24,663(1,985)$ & $14,277(1,582)$ & $<0.001$ \\
\hline Medical expenses & $19,835(1,948)$ & $11,462(1,511)$ & $<0.001$ \\
\hline Prescription drugs & $4,828 \quad(243)$ & $2,816 \quad(317)$ & $<0.001$ \\
\hline \multicolumn{4}{|c|}{ Kidney disease-related health expenses (\$), mean (SE) } \\
\hline Total expenses & $6,999 \quad(943)$ & $5,504(1,084)$ & 0.287 \\
\hline Medical expenses & $6,414 \quad(922)$ & $5,200(1,070)$ & 0.376 \\
\hline Prescription drugs & $585 \quad(90)$ & $304 \quad(64)$ & 0.011 \\
\hline \multicolumn{4}{|c|}{$\begin{array}{l}\text { a Nephrotic exposure is defined as medications associated with inducing acute tubular nephritis and/or tubular toxicity. Study population is from MEPS, } 2006-2012 \\
(n=809 \text {, weighted } n=14,665,892) \text {. } \\
C K D=\text { chronic kidney disease; } E D=\text { emergency department; MEPS = Medical Expenditure Panel Survey; SE=standard error. }\end{array}$} \\
\hline
\end{tabular}

those exposed to nephrotoxic medications versus nonexposure for demographic characteristics, medication prescribing patterns, and the likelihood of nephrotoxic medication exposure. Significant differences were also observed for all-cause health services utilization and total all-cause medical, prescription medication, and overall health expenses. Although significant differences were shown for all-cause exposure to nephrotoxic medication exposure versus nonexposure, no significant differences existed for specific health services utilization or health expenses specific to kidney disease.

Individuals with predialysis CKD are at greater risk for disease progression and poor outcomes when exposed to renal insults; therefore, preserving renal function is essential to avoiding ESRD. Preventing drug-induced nephrotoxicity requires close attention to choosing drugs, dosing, evaluating drug combinations, and patient outcomes when making unavoidable potentially nephrotoxic drug choices or where conflicting information about nephrotoxicity exists. ${ }^{31}$ This analysis of a nationally representative sample of U.S. adults with predialysis CKD shows that approximately $72 \%$ of these adults (weighted frequency approximating 10.5 million individuals) were prescribed 1 or more medications associated with acute tubular nephritis and/or acute tubular toxicity. This proportion seems out of step with the call to preserve renal function in the predialysis CKD patient population. Moreover, it is concerning to see that more than $50 \%$ of the population are being prescribed 2 or more potentially nephrotoxic medications. Although this study could not evaluate whether exposure to nephrotoxic medications resulted in kidney damage, the large proportions in this study cohort with an exposure suggest that many in the predialysis CKD population are at risk for tubular kidney damage. This study limited nephrotoxicity medication exposure to 1 underlying mechanism and, in so doing, may have underrepresented the scope of exposure to overall druginduced toxic insults in predialysis CKD. While the MULTUM drug classification system allowed this study to be fairly comprehensive, we may not have included the total universe of medications associated with a risk for inducing acute tubular nephritis and/or tubular toxicity. Future research could focus on further quantifying the scope of nephrotoxic medication exposure and incidence for, and amount of, kidney damage in the predialysis CKD population.

Older individuals and individuals with comorbid conditions appear vulnerable to exposure to nephrotoxic medications. This is concerning because estimated GFR declines with age, ${ }^{32}$ and elders with higher comorbidity are at greater risk for poor outcomes after an AKI. ${ }^{33}$ This study also found regional differences, similar to regional differences in the REGARDS study. ${ }^{34}$ 
TABLE 4 Association Between Health Expenses and Prescribed Medications Associated with Nephrotoxic Exposure in Adults with Predialysis $C K D^{a}$

\begin{tabular}{l|c|c|c}
\hline & \multicolumn{1}{|c|}{$\begin{array}{c}\text { Estimated Log } \\
\text { Coefficient (SE) } \\
\text { (Nephrotoxic Exposure } \\
\text { vs. Nonexposure) }\end{array}$} & $\begin{array}{c}\text { Change } \\
\text { in \% }\end{array}$ & $P$ Value \\
\hline All-cause expenses & \multicolumn{4}{|l}{} \\
\hline Total & $0.26(0.08)$ & +29.7 & 0.003 \\
\hline Medical & $0.21(0.10)$ & +23.4 & 0.036 \\
\hline Prescription drugs & $0.45(0.09)$ & +56.8 & $<0.001$ \\
\hline
\end{tabular}

Kidney disease-related expenses

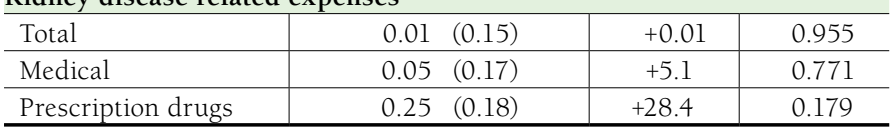

a Nephrotic exposure is defined as medications associated with inducing acute tubular nephritis and/or tubular toxicity. Study population is from MEPS, 2006-2012 $(n=809$, weighted $n=14,665,892)$.

${ }^{b}$ Linear regression with log link and gamma distribution was applied to examine association between health care expenses and nephrotoxic medication exposure after adjusting for age, perceived health status, insurance coverage, region, and comorbidity.

$C K D=$ chronic kidney disease; $M E P S=$ Medical Expenditure Panel Survey; SE $=$ standard error.

Regional differences point to possible disparities in care that need further investigation.

Significantly greater association was found between allcause (defined as all ICD-9-CM diagnosed medical conditions) health services utilization and health expenses for those exposed to nephrotoxic medications but was not found for health services utilization and expenses specific for kidney disease. Compared with the nonexposure group, significant increases in prescription drug, medical, and total expenses were observed in the nephrotoxic exposure group. Moreover, the increase in prescription drug expense was greater than medical and total expenses. This trend suggests that subjects exposed to nephrotoxic medications were more likely to use additional prescription drugs and medical care, which had an impact on health care expenses. The average prescription drug expense was much lower than the medical expenses, so the relative impact of the increase in the prescription drug expense was greater than the increase in the medical expense. Additionally, the prescription drug expense only accounted for $20 \%$ of total expenses. Thus, the effect of drug expense on the total expenses was not as great as the medical expense.

Few recent studies quantify health services utilization and costs in the predialysis CKD population. Vupputuri et al. (2014) found 2 to 4 times significantly greater costs with progression to stage 3 or 4 kidney disease among those with CKD and type 2 diabetes. ${ }^{35}$ These findings highlight economic implications, particularly for those with comorbid chronic disease, and the importance for calls for strategies to preserve renal function and minimize disease progression. Medications associated with renal tubular harm may account for the economic burden of predialysis CKD. Future research could further disentangle economic factors from other mediating factors in predialysis CKD (e.g., advanced age, comorbidity, severity of illness, or experiencing a drug-induced nephrotoxic insult) that contribute to the overall burden of predialysis CKD.

\section{Limitations}

There are several limitations to this study. The observational research design limited our ability to determine a cause and effect relationship between a nephrotoxic exposure and increased health services utilization and expenses. MEPS data do not provide laboratory information thereby limiting the ability to determine the severity of renal dysfunction or categorize the stage of predialysis CKD based on severity of disease. This study could not determine the presence of metabolic imbalances that would place an individual at greater risk for drug toxicity (e.g., hyperkalemia, hypokalemia, hypomagnesemia, or hypercalcemia ${ }^{36}$ or evaluate whether the cause of increased expenditures and utilization for nephrotoxic medication exposure resulted in a tubulopathy or reduced renal function or led to acute kidney disease or progression of kidney disease. MEPS data do not extend beyond a 2-year interview window, which limited the assessment of long-term individual patient outcomes.

This study did not assess individual disease progression to ESRD over the 5 interview rounds. Although drug dosing plays a role in nephrotoxicity, this analysis did not evaluate medication dose. This study only assessed medications associated with 1 underlying mechanism of kidney damage (e.g., tubular toxicity). Although the study controlled for comorbidity by using the CCI and assessed nephrotoxic medication exposure by coexistent chronic disease, this study could not evaluate whether the exposure was unavoidable or if a another prescribed medication would lower the risk of a drug-induced toxic exposure.

Additionally, the MEPS self-reported survey data may result in recall bias. However, the time span between the 5 interview intervals, rigorous data collection, and interview methods employed by MEPS field interviewers, as well as approaches taken to encode data, were designed to mitigate recall bias as a source of systematic error. ${ }^{37}$ Finally, although matching would add a level of rigor by allowing us to compare outcomes between the exposure and nonexposure groups to estimate the effect of nephrotoxic exposure while reducing bias, we did not perform matching in this study. One of the benefits of the MEPS data is the ability to apply weights and derive nationally representative estimates. Matching would have necessitated losing a good number of the exposure subjects. In this study, the number of nonexposed and exposed subjects were 250 and 559, respectively. Losing about 300 subjects would bias the 


\section{Nephrotoxic Medication Exposure in U.S. Adults with Predialysis Chronic Kidney Disease: Health Services Utilization and Cost Outcomes}

representativeness of the sample. To control for confounding, multivariable linear regression models were employed to assess the association between nephrotoxic medication exposure and health care utilization and expenses while controlling for demographic characteristics, socioeconomic status, and health status characteristics.

\section{Conclusions}

The growing economic and public health burden of predialysis CKD requires vigilance in preventing untoward patient outcomes that could add to that burden. Heightened awareness among medication prescribers is needed to identify those with predialysis CKD at risk for being prescribed medications that could be nephrotoxic and contribute to declining renal function. Particular attention to prescribing should be made for older patients, those with comorbid chronic conditions, and when choosing medication treatment plans that may include diuretics, proton pump inhibitors, NSAIDs, and anti-infective agents, since these agents are associated with increased cost of care and service utilization in CKD patients.

\section{Authors}

MARY LYNN DAVIS-AJAMI, PhD, Virginia Commonwealth University School of Nursing, Richmond, Virginia; JEFFERY C. FINK, MD, MS, University of Maryland Medical System, Baltimore, Maryland; and JUN WU, PhD, Presbyterian College School of Pharmacy, Clinton, South Carolina.

AUTHOR CORRESPONDENCE: Mary Lynn Davis-Ajami, PhD, Assistant Professor, Adult Health and Nursing Systems, School of Nursing, Virginia Commonwealth University, Richmond, VA 23298. Tel.: 804.828.3216; Fax: 804.828.7743; E-mail: mldavisajami@vcu.edu.

\section{DISCLOSURES}

There was no grant or intramural funding for this research. The authors have no conflicts of interest, financial or otherwise, to disclose.

Study concept and design were primarily contributed by Davis-Ajami, along with Fink and Wu. Davis-Ajami took the lead in data collection, along with $\mathrm{Wu}$, and data interpretation was performed by David-Ajami, $\mathrm{Wu}$, and Fink. All authors participated in manuscript preparation and revision.

\section{ACKNOWLEDGMENTS}

The authors acknowledge Jing-Jing Chen, who contributed to data management for this research.

\section{REFERENCES}

1. National Kidney Foudation. KDIGO 2012 clinical practice guideline for the evaluation and management of chronic kidney disease. Kidney Int. Suppl. 2013;3(1):1-150. Available at: http://www.kdigo.org/clinical_practice_guidelines/pdf/CKD/KDIGO_2012_CKD_GL.pdf. Accessed June 22, 2016.
2. National Guideline Clearinghouse. Guideline summary. KDIGO 2012 clinical practice guideline for the evaluation and management of chronic kidney disease. August 24, 2015. Updated April 15, 2016. Available at: http://www.guideline. gov/content. aspx?id=46510\&search=kdigo $+2012+$ clinic al+practice+guideline + for + the + evaluation + and + management + of + chronic + kid ney+disease\#tiptop. Accessed June 22, 2016.

3. Choudhury D, Ahmed Z. Drug-associated renal dysfunction and injury. Nat Clin Pract Nephrol. 2006;2(2):80-91.

4. Naughton CA. Drug-induced nephrotoxicity. Am Fam Physician. 2008;78(6):743-50.

5. Waller DG. Drugs and the kidney: more than a question of dose. Br J Clin Pharmacol. 2007;64(6):719-21.

6. National Institute of Diabetes and Digestive and Kidney Diseases. Kidney disease statistics for the United States. Available at: http://www.niddk.nih. gov/health-information/health-statistics/Pages/kidney-disease-statisticsunited-states.aspx\#3. Accessed June 22, 2016

7. Honeycutt AA, Segel JE, Zhuo X, Hoerger TJ, Imai K, Williams D. Medical costs of CKD in the Medicare population. J Am Soc Nephrol. 2013;(9):1478-83.

8. United States Renal Data System. 2015 USRDS annual data report: epidemiology of kidney disease in the United States. National Institutes of Health, National Institute of Diabetes and Digestive and Kidney Diseases, Bethesda, MD. 2015. Available at: https://www.usrds.org/adr.aspx. Accessed June 22, 2016.

9. Centers for Disease Control and Prevention. Chronic Kidney Disease (CKD) Surveillance Project. Prevalence of CKD in the health care system population. Available at: https://nccd.cdc.gov/ckd/detail.aspx?Qnum=Q380 Accessed June 22, 2016.

10. Aspden P, Wolcott J, Bootman JL, Cronenwett LR, eds. Preventing Medication Errors. Washington, DC: National Academies Press; 2007.

11. Leape LL. IOM medical error figures are not exaggerated. JAMA. 2000;284(1):95-97.

12. Gyllensten H, Hakkarainen KM, Hägg S, et al. Economic impact of adverse drug events - a retrospective population-based cohort study of 4970 adults. PloS One. 2014:9(3):e92061.

13. Wang X, Bonventre JV, Parrish AR. The aging kidney: increased susceptibility to nephrotoxicity. Int J Mol Sci. 2014;15(9):15358-76.

14. Ishani A, Xue JL, Himmelfarb J, et al. Acute kidney injury increases risk of ESRD among elderly. J Am Soc Nephrol. 2009;20(1):223-28.

15. United States Renal Data System. USRDS 2007 annual data report. National Institute of Diabetes and Digestive and Kidney Diseases, National Institutes of Health, Bethesda, MD. 2007. Available at: https://www.usrds. org/atlas07.aspx. Accessed July 1, 2016.

16. Chawla LS, Eggers PW, Star RA, Kimmel PL. Acute kidney injury and chronic kidney disease as interconnected syndromes. N Engl J Med. 2014;371(1):58-66.

17. Menon S, Kirkendall ES, Nguyen H, Goldstein SL. Acute kidney injury associated with high nephrotoxic medication exposure leads to chronic kidney disease after 6 months. J Pediatr. 2014;165(3):522-27.

18. McGregor TL, Jones DP, Wang L, et al. Acute kidney injury incidence in noncritically ill hospitalized children, adolescents, and young adults: a retrospective observational study. Am J Kidney Dis. 2016;67(3):384-90.

19. Agency for Healthcare Research and Quality. Medical Expenditure Panel Survey (MEPS). Survey background. August 21, 2009. Available at: http://meps. ahrq.gov/mepsweb/about_meps/survey_back.jsp. Accessed June 22, 2016.

20. Healthcare Cost and Utilization Project (HCUP). Clinical Classifications Software (CCS) for ICD-9-CM. Agency for Healthcare Research and Quality, Rockville, MD. May 2016. Available at: http://www.hcup-us.ahrq.gov/ toolssoftware/ccs/ccs.jsp. Accessed June 22, 2016.

21. Elixhauser A, Steiner C, Palmer L. Clinical Classifications Software (CCS), 2015. Agency for Healthcare Research and Quality. March 2016. Available at: http://www.hcup-us.ahrq.gov/toolssoftware/ccs/ CCSUsersGuide.pdf. Accessed June 22, 2016. 
22. Santos WJ, Zanetta DM, Pires AC, Lobo SM, Lima EQ, Burdmann EA. Patients with ischaemic, mixed and nephrotoxic acute tubular necrosis in the intensive care unit-a homogeneous population? Crit Care. 2006;10(2):R68.

23. Agency for Healthcare Research and Quality. Medical Expenditure Panel Survey (MEPS). MEPS HC-068: Multum Lexicon Addendum Files to MEPS Prescribed Medicine Files 1996-2001. C. Technical Information; 1.0 Data File Contents. Available at: http://meps.ahrq.gov/mepsweb/data_stats/download_data/pufs/h68/h68doc.shtml. Accessed June 22, 2016.

24. Cerner Multum. Lexicon Plus. August 24, 2015. Available at: http://multum.com/lexicon.html. Accessed June 22, 2016.

25. Soni A. Expenditures for the top five therapeutic classes of outpatient prescription drugs, Medicare beneficiaries, age 65 and older, 2007. Statistical Brief \#280. February 2010. Agency for Healthcare Research and Quality, Rockville, MD. Available at: https://meps.ahrq.gov/data_files/publications/st280/stat280.pdf. Accessed June 22, 2016

26. Deyo RA, Cherkin DC, Ciol MA. Adapting a clinical comorbidity index for use with ICD-9-CM administrative databases. J Clin Epidemiol. 1992;45(6):613-19.

27. Chu YT, NG YY, Wu SC. Comparison of different comorbidity measures for use with administrative data in predicting short-and long-term mortality. BMC Health Serv Res. 2010;10:140.

28. Agency for Healthcare Research and Quality. Medical Expenditure Panel Survey (MEPS). Using appropriate price indices for analyses of health care expenditures or income across multiple years. Available at: https://meps. ahrq.gov/about_meps/Price_Index.shtml. Accessed June 22, 2016.
29. U.S. Bureau of Labor Statistics. Consumer Price Index. Available at: http://www.bls.gov/cpi/home.htm. Accessed June 22, 2016.

30. Manning WG, Mullahy J. Estimating log models: to transform or not to transform? J Health Econ. 2001;20(4):461-94.

31. Bicalho MD, Soares DB, Botoni FA, Reis AM, Martins MA. Druginduced nephrotoxicity and dose adjustment recommendations: agreement among four drug information sources. Int J Environ Res Public Health. 2015;12(9):11227-40.

32. Malmgren 1, McGuigan FE, Berglundh S, Westman K, Christensson A, Âkesson K. Filtration rate and its association with mortality and comorbidity over 10 years in elderly women. Nephron. 2015;130(4):245-55.

33. Hamzic-Mehmedbasic A, Rebic D, Balavac M, Muslimovic A, Dzemidzic J. Clinical analysis of etiology, risk factors and outcome in patients with acute kidney injury. Mater Sociomed. 2015;27(2):70-74.

34. Tanner RM, Gutiérrez OM, Judd S, et al. Geographical variation in CKD prevalence and ESRD incidence in the United States: results from the Reasons for Geographic and Racial Differences in Stroke (REGARDS) study. Am J Kidney Dis. 2013;61(3):395-403.

35. Vupputuri S, Kimes TM, Calloway MO, et al. The economic burden of progressive chronic kidney disease among patients with type 2 diabetes. J Diabetes Complications. 2014;28(1):10-16.

36. Perazella MA. Renal vulnerability to drug toxicity. Clin J Am Soc Nephrol. 2009;4(7):1275-83

37. Zuvekas SH, Olin GL. Validating household reports of health care use in the Medical Expenditure Panel Survey. Health Serv Res. 2009;44(5 Pt 1): 1679-700. 
Nephrotoxic Medication Exposure in U.S. Adults with Predialysis

Chronic Kidney Disease: Health Services Utilization and Cost Outcomes

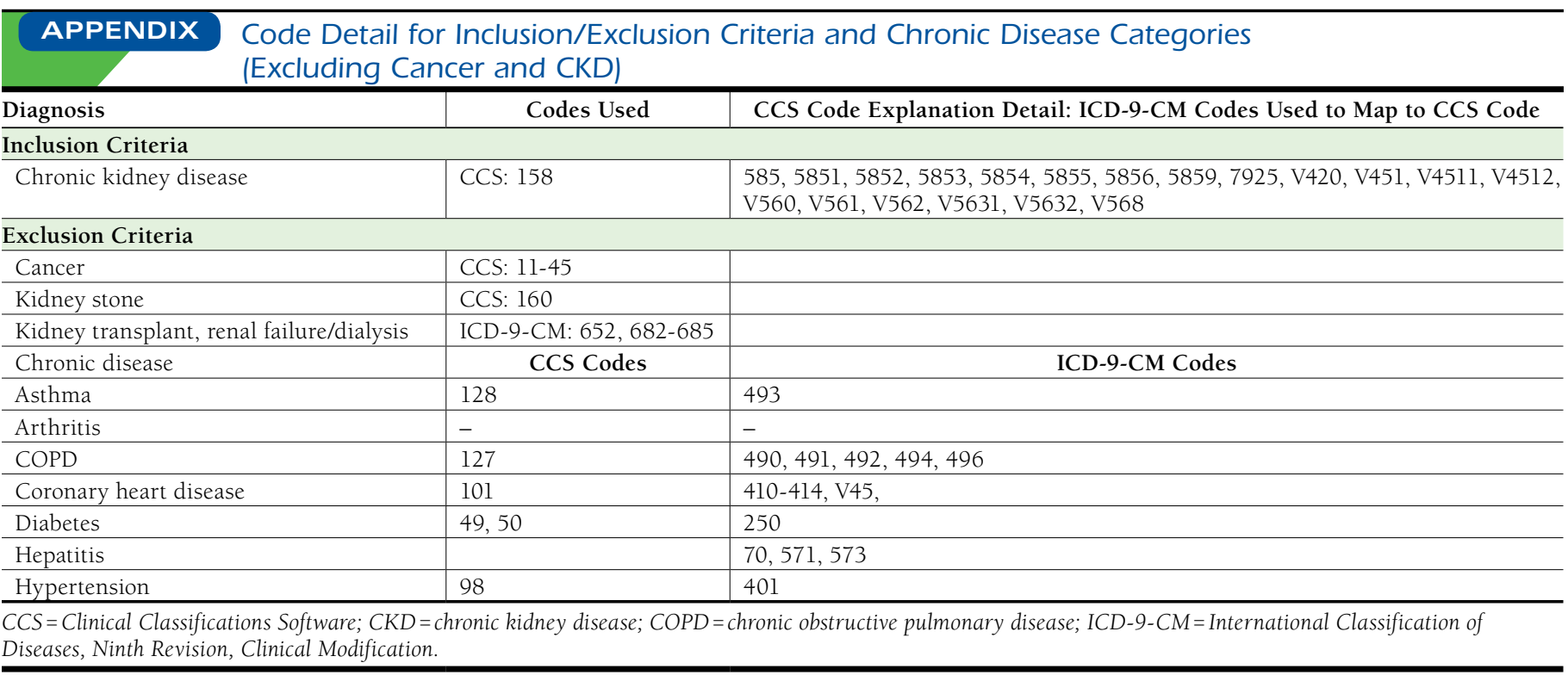

